\title{
3 Die Quellen des Jalkut Schimoni Zwölfprophetenbuch
}

Bereits Leopold Zunz ging davon aus, dass die Quellenangaben, die in der Editio Princeps des Jalkut Schimoni im Fließtext und im Druck Venedig am Seitenrand notiert sind, vom Verfasser selbst stammen: ${ }^{1}$

\begin{abstract}
Eine der wichtigsten Arbeiten bei der Zusammenstellung des Jalkut war die Bezeichnung der Quellen. Diese ist unstreitig von dem Verfasser selbst ausgegangen. Kein Jüngerer wäre im Stande gewesen, jedes Fragment in den Hagada‘s aufzusuchen, die ähnlichen Midraschim sorgfältig zu unterscheiden, die Characteristik der Benennung mit solcher Treue zu beobachten, oder sich vor der Einschwärzung neuerer Hagada's zu hüten. Nach einer alten Bibelausgabe sollte man fast vermuthen, dass der Verfasser die Quellen im Texte selbst angegeben, und diese erst durch Abschreiber an den Rand geraten seien (In der ersten Bombergischen Ausgabe der Rabbinischen Bibel (Venedig 1517) liest man zu Esra und der Chronik einen פירוש השמוני, aus JalkutExcerpten bestehend).
\end{abstract}

Im Folgenden wird ausgewertet, welche Quellen für den Kommentar des Jalkut Schimoni zum Zwölfprophetenbuch verwendet wurden und wie diese Quellen zu einem fortlaufenden Kommentar kombiniert wurden. In fast allen Fällen, mit Ausnahme von Midrasch Jona und Midrasch Samuel, war der Autor des Jalkut gezwungen, auf teils beiläufige Auslegungen zu den prophetischen Büchern zurückzugreifen und diese aus dem Kontext herauszulösen und zu einer funktionierenden Auslegung umzugestalten. Dies konnte dazu führen, dass Auslegungen umgruppiert werden mussten, damit sie als Kommentierung eines Prophetenverses passten.

\section{Jalkut Schimoni Hosea}

Mit 14 Kapiteln und knapp 47 hebräischen Textseiten ist die Kommentierung des Jalkut Schimoni zu Hosea eine der längsten Kommentare innerhalb des Jalkut zum Zwölfprophetenbuch.

1 Leopold Zunz, Die gottesdienstlichen Vorträge der Juden. Ein Beitrag zur Altertumskunde und biblischen Kritik zur Literatur- und Religionsgeschichte (Berlin: A. Asher, 1832), 314. 
Tab. 1: Anzahl der in Jalkut Schimoni Hosea verwendeten Quellen

\begin{tabular}{llll}
\hline Quelle & Anzahl & Quelle & Anzahl \\
\hline bTalmud $^{2}$ & 64 & Midrasch Psalmen $^{3}$ & 2 \\
Pes. de-Rav Kahana $^{4}$ & 17 & Avot de-Rabbi Natan $^{5}$ & 1 \\
Genesis Rabba $^{6}$ & 15 & Canticum Rabba $^{7}$ & 1 \\
Pesiqta Rabbati $^{8}$ & 10 & Dtn Rabba $^{9}$ & 1 \\
Sifre Dtn $^{10}$ & 7 & Midrasch Samuel $^{11}$ & 1 \\
Tanchuma $^{12}$ & 7 & Rut Rabba $^{13}$ & 1 \\
Levitikus Rabba $^{14}$ & 6 & Seder Olam $^{15}$ & 1 \\
Jelamdenu $^{15}$ & 5 & Sifre Numeri $^{16}$ & 1 \\
Threni Rabba $^{17}$ & 5 & Tanchuma $^{18}$ & 1 \\
Mekhilta $^{19}$ & 4 & Unbekannt $^{1}$ & 1 \\
\hline
\end{tabular}

2 In den folgenden Auflistungen aller Quellentexte des Jalkut zum Zwölfprophetenbuch, werden mit (v) die Quellen gekennzeichnet, auf die verwiesen wird: bAZ 4a; bAZ 18b; bBB 8a; bBB 14b (v); bBB 25a; bBB 89b; bBer 6b; bBer 7a; bBer 32a; bBer 35b; bBer 43b; bChul 92a; bGit 7a; bGit 31b; bGit 60b; bJeb 102b; bJoma 22b; bJoma 38b; bJoma 76b; bJoma 86a-b; bKet 64b-65a; bMak 10a; bMak 10b-11a (v); bMeg 13b (v); bMeg 14b (v); bMeg 17b-18a; bMeg 25b; bMeg 25b (v); bMQ 17a; bNazir 23a; bPes 52b; bPes 87a; bPes 87a-b; bPes 87b; bPes 87b-88a (v); bPes 88a; bQid 13a; bQid 36a (v); bQid 70a; bQid 70b; bRH 31a; bRH 31a (v); bSanh 102a; bSanh 102b; bSanh 106a; bSanh 111a; bSanh 38b; bSanh 63b; bSchab 119b; bSchab 145b; bSchab 55a; bSota 37a; bSota 42a; bSota 47a-b; bSuk 49b; bSuk 52b; bTaan 5b (v); bTaan 29b-30a; bTaan 5a; bTaan 6b.

3 MidrPs 31,8 (v); MidrPs 45,3.

4 PRK 1,8 (v); PRK 5,10; PRK 5,8; PRK 12,4; PRK 9,1 (v); PRK 15,5; PRK 15,8-9 (v); PRK 17,8 (v) wird zweimal als Verweis angeführt; PRK 24,9; PRK 24,10; PRK 24,12; PRK 24,13; PRK 24,15; PRK 24,17; PRK 24,18; PRK 24,19.

5 ARN A 4.

6 GenR 19,9; GenR 28,7 (v); GenR 29,3; GenR 38,6; GenR 46,1; GenR 48,6 (v); GenR 56,1 (v); GenR 59,9; GenR 70,20; GenR 78,2; GenR 80,1 (v); GenR 80,2; GenR 84,19; GenR 86,1; GenR 86,2.

7 CantR 1,4 (v).

8 PR 3,4; PR 4,2 (v); PR 5,14; PR 11,4; PR 24,11; PR 26,1/2 (v); PR 33,7; PR 44,3-4; PR 44,5; PR 44,9.

9 DtnR 2,19.

10 SDtn $\S 47$ (v); SDtn § 305; SDtn § 306 wird zweimal, SDtn $\S 306$ (v) dreimal als Verweis angeführt.

11 MidrSam 13,4 (v).

12 TanB bechuqqotai 2; TanB balak 22 (v); TanB bereschit 20 (v); TanB chuqqot 10 (v); TanB lekh 21;

TanB schmot 7; TanB wa-jera 22.

13 RutR 1,2 (v).

$14 \operatorname{LevR}$ 1,2; LevR 1,14; LevR 7,3; LevR 12,5 (v); LevR 19,5 (v); LevR 24,4 (v).

15 Seder Olam 20.

16 SNum $\S 131$.

17 ThrR 1,3; ThrR 2,2; ThrR 2,3; ThrR pet. 6; ThrR pet. 22 (v).

18 Tan chuqqat 6 (v).

19 Mek beschal 5; Mek beschal 6 (v); Mek beschal 7; Mek beschal 7 (v). 


\begin{tabular}{llll}
\hline Quelle & Anzahl & Quelle & Anzahl \\
\hline jTalmud $^{20}$ & 2 & & \\
\hline
\end{tabular}

Zur Auslegung von Hos 5,7 wird eine Quelle verwendet, die nicht zu identifizieren ist. Diese Quelle ergänzt die Auslegung aus TanB schemot 7. Zu Hos 13,12 wird als Quelle PR 44,2 mit einem Gleichnis verwendet, das allerdings in PR 44,2 nicht mehr belegbar ist. ${ }^{21}$ Auf insgesamt 39 Quellenstücke wird ausschließlich oder textergänzend ${ }^{22}$ verwiesen. Die Quellenauswertung ergibt die folgenden Werte: ${ }^{23}$

- Babylonische Quellen: 64

_ Palästinische Quellen: 88

- Unbekannt: 1

\section{Jalkut Schimoni Joel}

Jalkut Schimoni Joel umfasst, wie das biblische Buch Joel, vier Kapitel und hat knapp 13 hebräische Textseiten.

Tab. 2: Anzahl der in Jalkut Schimoni Joel verwendeten Quellen

\begin{tabular}{llll}
\hline Quelle & Anzahl & Quelle & Anzahl \\
\hline bTalmud $^{24}$ & 7 & Midrasch Psalmen & 25 \\
Mekhilta $^{26}$ & 3 & Pes. de-Rav Kahana & 2 \\
Tanchuma $^{28}$ & 3 & Sifre Dtn & 2 \\
\hline
\end{tabular}

20 jPea 2,6; jScheq 5,7(6).

21 Siehe Pesiqta Rabbati. A Synoptic Edition of Pesiqta Rabbati based upon all extant Manuscripts and the Editio Princeps, hg. Rivka Ulmer, Bd. 2 (Lanham: University Press of America, 2009), 984-985.

22 Das heißt die Quelle wird anzitiert und darauf wird diese Auslegung abgebrochen und es folgt ein Verweis.

23 Diese Listung zeigt eine weitere Auswertung der Quellenstatistik. Diese ist nach „palästinischen“ und „babylonischen“ sowie unbekannten Quellen aufgeteilt. Die gleiche Aufteilung gilt für alle weiteren Graphiken dieses Kapitels.

24 bBQ 119a (v); bNed 39b; bSuk 52a; bSuk 52a (v); bTaan 5a; bTaan 6a; bTaan 12b.

25 MidrPs 6,6; MidrPs 80,1.

26 Mek beschallach 2 wird zweimal verwendet, Mek pischa 12 (v).

27 PRK 24,11; PRK 24,3.

28 TanB balak 26; TanB bereschit 12; TanB wa-jiqra 1.

29 SDtn $\S 49$. 


\begin{tabular}{llll}
\hline Quelle & Anzahl & Quelle & Anzahl \\
\hline jTalmud $^{30}$ & 2 & Seder Elijahu Zuta & 1 \\
Levitikus Rabba $^{32}$ & 2 & & \\
\hline
\end{tabular}

Auf insgesamt 4 Quellenstücke wird ausschließlich oder textergänzend verwiesen. Die Quellenauswertung ergibt die folgenden Werte:

- Babylonische Quellen: 7

_ Palästinische Quellen: 16

\section{Jalkut Schimoni Amos}

Jalkut Schimoni Amos umfasst neun Kapitel und knapp 28 hebräische Textseiten.

Tab. 3: Anzahl der in Jalkut Schimoni Amos verwendeten Quellen

\begin{tabular}{llll}
\hline Quelle & Anzahl & Quelle & Anzahl \\
\hline bTalmud $^{33}$ & 37 & Dtn Rabba & \\
Genesis Rabba $^{35}$ & 7 & Jelamdenu & 1 \\
Tanchuma $^{36}$ & 5 & jTalmud $^{37}$ & 1 \\
Levitikus Rabba $^{38}$ & 3 & Mekhilta $^{39}$ & 1 \\
Midrasch Psalmen $^{40}$ & 3 & Midrasch Samuel $^{41}$ & 1 \\
\hline
\end{tabular}

30 jBer 9,1; jTaan 2,1.

31 SEZ 1.

32 LevR 31,9; LevR 35,12.

33 bAZ 4a; bBB 90b; bBer 3a (v); bBer 4b (v); bBer 6a; bBer 23a; bBer 56b; bBM 59a; bChag 4b; bChag 5b; bChag 12b; bChul 59b-60a; bChul 87a; bJoma 86b; bKer 6b; bMak 24a (v); bMQ 15b; bMQ 20a; bMQ 16b (v); bMQ 25a; bMQ 25b; bMQ 28b; bNed 38a (v); bNid 65a; bPes 49a; bPes 87b (v); bRH 31a (v); bSanh 7a; bSanh 96b-97a; bSanh 98b; bSanh 98b (v); bSchab 10a; bSchab 32b-33a; bSchab 62b; bSchab 77b; bSchab 138b-139a; bTaan 6b.

34 DtnR 10,2.

35 GenR 23,7; GenR 25,2; GenR 25,3 (v); GenR 31,3; GenR 49,2; GenR 71,2; GenR 93,5.

36 TanB tetse 4 (v); TanB debarim 5; TanB nitsabim 4 wird zweimal angeführt; TanB toldot 2.

37 jAZ 1,1.

38 LevR 5,3; LevR 33,2; LevR 33,3.

39 Mek bachodesch 4.

40 MidrPs 18,11; MidrPs 18,11 (v); MidrPs 25,13.

41 MidrSam 14,1. 


\begin{tabular}{llll}
\hline Quelle & Anzahl & Quelle & Anzahl \\
\hline Pes. de-Rav Kahana & 3 & Panim Acherot Ester & 1 \\
Seder Olam $^{44}$ & 3 & Pirk. de-Rab. Eliezer $^{45}$ & 1 \\
Sifre Dtn $^{46}$ & 3 & Threni Rabba $^{47}$ & 1 \\
Avot de-Rabbi Natan $^{48}$ & 1 & & \\
\hline
\end{tabular}

Im Zuge der Quellenauswahl sticht in Jalkut Amos in Kapitel 4 die Quelle jAZ 1,1 hervor. Innerhalb des Zwölfprophetenbuches im Jalkut Schimoni wird auch der Jerusalemer Talmud immer wieder als Textressource verwendet, jedoch in überschaubaren, tendenziell kurzen Quellenstücken. An der angeführten Stelle handelt es sich um eine Auslegung, die über vier Textseiten fortlaufend zitiert wird und so Auslegungen $\mathrm{zu}$ Am 4,4 und Am 4,5 konstruiert. In diesem Umfang stellt diese Textpassage eine Besonderheit dar.

Der Jalkut legt zu Amos die Verse fortlaufend in der Folge der biblischen Bücher aus. An mehreren Stellen in Amos wird diese Auslegungsfolge jedoch gebrochen. Am 8,7 wird in Kapitel 6 ausgelegt. Diese Auslegung wird gesondert und als Teil einer neuen Quelle (bBB 90b) am Ende des Kapitels zitiert. Diese Vorgehensweise unterstützt die These, dass der Autor eine inhaltlich verbundene Auslegungsstrategie verfolgt und deshalb auch die Chronologie brechende Auslegungen in Kauf nimmt, sofern diese inhaltliche Ergänzungen zulassen..$^{49}$ Das gleiche Prinzip wiederholt sich mit der Auslegung zu Am 9,1, die in Kapitel 7 gegeben wird. Dafür wird die Quelle LevR 33,3 eingefügt, folgend auf eine Auslegung zu Am 7,8, worauf sich dann eine weitere Auslegung zu Am 7,7 anschließt. Weitere Beispiele für eine nicht fortlaufende Auslegung finden sich in Am 1: Hier wird Am 2,6 nach Am 1,2 ausgelegt, worauf dann Am 1,9 folgt. Eine weitere Auslegung zu Am 6,4 findet sich nicht folgend auf die bereits gezeigten Auslegungen zum gleichen Vers, sondern nach Am 6,7. Auf diese Auslegung von Am 6,4 folgen dann zwei zu Am 6,6, eine weitere zu Am 6,4, dann Am 6,7. Zum Schluss des Kapitels 6 folgt die Auslegung zu Am 8,7. Zum Ende des Kapitels 8 wird nach den Auslegungen zu Am 8,12 noch einmal auf eine weitere Auslegung zu Am 8,11 verwiesen. Auf insgesamt 17 Quellenstücke wird ausschließlich oder textergänzend verwiesen. Die statistische Quellenauswertung ergibt folgendes Bild:

42 PRK 5,9; PRK 13,15 (v); PRK 24,1.

43 PAB Est 1,1.

44 Seder Olam 20 (v); Seder Olam 22 (v); Seder Olam 28.

45 PRE 37-38.

$46 \operatorname{SDtn} \S 1$ (v); SDtn $\S 322 ; \operatorname{SDtn} \S 346$.

47 ThR pet. 2 (v).

48 ARN 3,6.

49 Siehe dazu Kapitel 5 ,Textkomposition im Jalkut Schimoni Zwölfprophetenbuch“. 
- Babylonische Quellen: 37

- Palästinische Quellen: 36

\section{Jalkut Schimoni Obadja}

Jalkut Schimoni Obadja ist, wie auch Jalkut Nahum, einer der kurzen Kommentare im Jalkut Schimoni; er umfasst 6 hebräische Textseiten.

Tab. 4: Anzahl der in Jalkut Schimoni Obadja verwendeten Quellen

\begin{tabular}{llll}
\hline Quelle & Anzahl & Quelle & Anzahl \\
\hline bTalmud $^{50}$ & 7 & Jelamdenu & 1 \\
Tanchuma $^{51}$ & 4 & Mekhilta & 1 \\
Genesis Rabba $^{53}$ & 2 & Pes. de-Rav Kahana & 1 \\
Unbekannt $^{55}$ & 2 & Seder Olam & 1 \\
\hline
\end{tabular}

In Jalkut Schimoni Obadja folgt auf die zusammenhängende Auslegung zu Ob 1,1114 die Auslegung zu Ob 1,12 und die Auslegung zu Ob 1,14, bevor eine weitere gesonderte Auslegung zu Ob 1,13 angeführt wird. Hier hat der Autor die Quelle (Jelamdenu) so belassen, da diese die gezeigte Struktur vorgab. An zwei Stellen bleiben die Verweise auf die verwendeten Quellen ungeklärt. ${ }^{57}$ Auf insgesamt 8 Quellenstücke wird ausschließlich oder textergänzend verwiesen. Die Quellenauswertung ergibt die folgenden Werte:

- Babylonische Quellen: 7

- Palästinische Quellen: 10

- Unbekannt: 2

50 bAZ 10a; bBB 123b; bBB 123b (v); bBQ 3b (v); bSanh 39b; bSanh 89a-b (v); bSanh 92a (v).

51 TanB tsaw 3-4 (v); TanB tsaw 4; TanB wa-jischlach 8 wird zweimal angeführt.

52 Mek pischa $12(\mathrm{v})$.

53 GenR 78,14; GenR 84,5.

54 PRK 3,16.

55 Es handelt sich in beiden Fällen um ungeklärte Verweise.

56 Seder Olam 20.

57 Zum einen ein Verweis auf den $\S 373$ und zum anderen auf den $\S 460$. 


\section{Jalkut Schimoni Jona}

Jalkut Schimoni Jona umfasst wie das biblische Buch vier Kapitel und hat knapp 13 hebräische Textseiten. Der Jonakommentar sticht deutlich durch seinen narrativen Charakter hervor. Eine strukturelle Besonderheit besteht bereits in der Auswahl der Quellen. Es werden sehr wenige Quellen kombiniert und fortlaufende narrative Passagen in Gänze beibehalten. Außerdem werden verhältnismäßig lange Passagen aus dem Jerusalemer Talmud gewählt, während in diesem Kommentar nur kurze Quellenstücke aus dem Babylonischen Talmud benutzt wurden.

Tab. 5: Anzahl der in Jalkut Schimoni Jona verwendeten Quellen

\begin{tabular}{llll}
\hline Quelle & Anzahl & Quelle & Anzahl \\
\hline Pirk. de-Rab. Eliezer $^{58}$ & 5 & jTalmud $^{59}$ & 4 \\
Bet ha-Midrasch $^{60}$ & 4 & Mekhilta $^{61}$ & 3 \\
bTalmud $^{62}$ & 4 & Unbekannt & $1^{63}$ \\
Genesis Rabba $^{64}$ & 4 & & \\
\hline
\end{tabular}

Der Jonakommentar verfolgt eine - zwar genau wie in den anderen prophetischen Büchern des Jalkut - fortlaufende aber sehr sprunghafte Auslegung. So werden in Jona lange fortlaufende Quellenstücke wiedergegeben und dadurch nur wenige verschiedene Verse aus dem biblischen Jonabuch im Jalkut ausgelegt. Es werden Jonaverse zitiert und als Belege für die auszulegenden Jonaverse verwendet. ${ }^{65}$ Dies suggeriert eine nicht fortlaufende Auslegung des Jonabuches. Die vielen Jonaverse, die zum Beleg der Auslegung dienen, unterstützen aber die narrative Struktur der Jalkutkommentierung zu Jona. Anzumerken ist weiterhin, dass der Kommentar zu Jona zwar genau wie die anderen Bücher im Jalkut zu den kleinen Propheten zu jedem biblischen Kapitel ein Kapitel im Jalkut anführt, ${ }^{66}$ jedoch zu Jona 2 lediglich zwei Paragra-

58 PRE 9; PRE 10 wird dreimal angeführt; PRE 43.

59 jBM 2,5 (v); jSuk 5,1; jTaan 2,1 wird zweimal angeführt.

60 BhM I,98; BhM I,99-100; BhM 1,100-1; BhM I,102.

61 Mek pischa 1 wird zweimal angeführt; Mek pischa $1(\mathrm{v})$.

62 bEr 19a (v); bNed 38a (v); bTaan 15a/16a; bRH 16b.

63 Es handelt sich hier um einen ungeklärten Verweis.

64 GenR 24,4 (v); GenR 37,4; GenR 55,7 (v); GenR 56,1 (v).

65 Jona 1,3 wird u.a. mit Jona 1,4 belegt. Jona 1,4 wird u.a. mit Jona 1,3; 1,12; 1,15; 1,16; 2,1; 2,2; 2,4; 2,6; 2,7; 2,9; 2,10 belegt. Jona 3,10 wird u.a. mit Jona 3,8 belegt.

66 Ausgenommen ist Nahum, dort liegt kein drittes Kapitel im Jalkut vor und Sacharja dort liegt kein siebtes Kapitel vor. 
phenverweise angibt und keine weiteren Ausführungen bietet. Auf insgesamt 8 Quellenstücke wird ausschließlich oder textergänzend verwiesen. Die Quellenauswertung ergibt folgendes Bild:

- Babylonische Quellen: 4

- Palästinische Quellen: 20

- Unbekannt: 1

\section{Jalkut Schimoni Micha}

Jalkut Micha umfasst wie das biblische Buch sieben Kapitel und hat knapp 23 hebräische Textseiten.

Tab. 6: Anzahl der in Jalkut Schimoni Micha verwendeten Quellen

\begin{tabular}{llll}
\hline Quelle & Anzahl & Quelle & Anzahl \\
\hline bTalmud $^{67}$ & 22 & Canticum Rabba $^{68}$ & 1 \\
Genesis Rabba $^{69}$ & 8 & Mekhilta $^{70}$ & 1 \\
Tanchuma $^{71}$ & 7 & Pes. de-Rav Kahana $^{72}$ & 1 \\
Jelamdenu $_{\text {Midrasch Psalmen }}^{74}$ & 3 & Pesiqta Rabbati $^{73}$ & 1 \\
jTalmud $^{76}$ & 2 & Pirk. de-Rab. Eliezer & 1 \\
Levitikus Rabba $^{78}$ & 2 & Seder Elijahu Zuta7 & 1 \\
\hline
\end{tabular}

67 bAZ 16b-17a; bBer 32a; bBer 56b (v); bBer 58a; bBer 7a; bChag 16a; bEr 101a; bEr 63b; bJoma 10a; bMak 23b-24a; bRH 17a; bRH 17a-b; bRH 31a (v); bSanh 102a; bSchab 63a (v); bSchab 139a; bSchab 63a; bSuk 49b; bSuk 52b; bSuk 52b (v); bTaan 11a.

68 CantR 8,9,3.

69 GenR 28,5; GenR 41,3 (v); GenR 42,2; GenR 48,10 (v); GenR 55,5; GenR 69,5; GenR 75,8; GenR 85,1.

70 Mek schira 8.

71 TanB berakha 3; TanB emor 13; TanB nitsabim 1; TanB toldot 19; TanB toldot 19 (v); TanB tsaw 1; TanB wa-jetse 24.

72 PRK 21,4.

73 PR 39,2.

74 MidrPs 1,20; MidrPs 22,7; MidrPs 22,7 (v).

75 PRE 48.

76 jTaan 2,1; jPea 1,1.

77 SEZ 21 (v).

78 LevR 24,4; LevR 24,4 (v).

79 Seder Olam 20 (v). 


\begin{tabular}{llll}
\hline Quelle & Anzahl & Quelle & Anzahl \\
\hline Sifre Dtn $^{80}$ & 2 & Sifra $^{81}$ & 1 \\
Unbekannt $^{82}$ & 2 & & \\
\hline
\end{tabular}

Eine neue Unregelmäßigkeit in der Auslegung zeigt sich in Jalkut Micha gleich mehrfach: Während die verwendeten Quellen an den betreffenden Stellen den benötigten Michavers nicht verwenden, verwenden sie einen gleichlautenden Vers eines anderen biblischen Buches. Dieser Vers wird im Jalkut unter „Micha“ geführt, um die Auslegung korrekt an diesen Stellen zu Ende zu führen. Konkret passiert dies in Kapitel 4 zur Auslegung von Mi 4,1. Diese Auslegung schließt mit SDtn § 28. Die Quelle SDtn $\S 28$ führt nicht Mi 4,1, sondern den gleichlautenden Vers Jes 2,2 an. Das Gleiche passiert zur Auslegung von Mi 4,3. Hier verwendet die Quelle bSchab 63a den Vers Jes 3,17, während im Jalkut dieses Quellenstück die Auslegung zu Mi 4,3 eröffnen soll und Jes 3,17 somit als Mi 4,3 anführt.

Besonders interessant wird es im Zuge der Auslegung zu Mi 5,6: Zunächst wird mit der Quelle GenR 75,8 eine Auslegung zu Mi 5,6 angeführt. Hier wird innerhalb der Quelle auch Mi 5,6 verwendet, zudem handelt es sich um einen größeren Versteil (Und es werden die Übrigen Jakobs sein [...] wie Tau von JHWH). Darauf folgt ein Verweis zu TanB toldot 19, Jalkut Richter § 62. Die Quelle selbst (TanB toldot 19) wird darauf noch einmal zitiert und ergänzt somit den „Verweis“ um das Quellenstück, welches nicht in $\S 62$ angeführt wird. Sowohl in der parallelen Stelle in Jalkut Richter, als auch in Jalkut Micha wird Mi 5,6 als Angabe zum Text (Und es werden die Übrigen Jakobs sein) angegeben. Außerdem wird in Jalkut Micha mit der Quelle die Auslegung für Mi 5,6 eröffnet, während in Jalkut Richter § 62 Mi 5,6 und der folgende Text der Quelle zur Auslegung von Jdc 6,12 verwendet wird. Es muss in Jalkut Micha aber als Mi 5,6 klassifiziert werden, um die folgenden Auslegungen in die passende Reihenfolge einzufügen.

In Kapitel 7 werden die Quellen bChag 16a und bTaan 11a miteinander verbunden. Dies ist möglich, weil in der Quelle bTaan 11a der Vers Hab 2,11 zitiert wird. Das Quellenstück bChag 16a endet mit diesem Vers. So wird bTaan 11a ohne Hab 2,11 nahtlos als fortlaufende Auslegung zu Mi 7,5 angeschlossen. Zu zwei Verweisen bleibt die verwiesene Quelle ungeklärt. ${ }^{83}$ Auf insgesamt 13 Quellenstücke wird ausschließlich oder textergänzend verwiesen. Die Quellenauswertung ergibt die folgenden Werte:

80 SDtn $\S 28 ; \operatorname{SDtn} \S 342$.

81 Sifra bechuq per. 4.

82 Es handelt sich in beiden Fällen um ungeklärte Verweise.

83 In beiden Fällen handelt es sich um Verweise auf Jesaja. Einmal ein Verweis zum $\S 55$ und ein Verweis auf $\S 391$. 
- Babylonische Quellen: 22

- Palästinische Quellen: 35

- Unbekannt: 2

\section{Jalkut Schimoni Nahum}

Jalkut Nahum ist der kürzeste unter den Kommentaren zu den zwölf kleinen Propheten. Er umfasst zwei Kapitel und vier hebräische Textseiten.

Tab. 7: Anzahl der in Jalkut Schimoni Nahum verwendeten Quellen

\begin{tabular}{llll}
\hline Quelle & Anzahl & Quelle & Anzahl \\
\hline bTalmud $^{84}$ & 3 & Jelamdenu & 1 \\
Genesis Rabba $^{85}$ & 3 & Seder Olam & \\
Tanchuma $^{87}$ & 3 & Sifre Dtn $^{88}$ & 1 \\
\hline
\end{tabular}

Eine Besonderheit von Jalkut Nahum ist, dass das biblische Buch Nahum drei Kapitel umfasst, der Jalkutkommentar aber nur die zwei ersten Kapitel behandelt und das letzte nicht berücksichtigt. In Jalkut Nahum Kapitel 1 wird zur Auslegung des Verses Nah 1,7 die Quelle TanB bemid 3 verwendet. Diese Quelle wird stark paraphrasiert. ${ }^{89}$ Auf insgesamt 2 Quellenstücke wird ausschließlich oder textergänzend verwiesen. Die statistische Quellenauswertung ergibt die folgenden Werte:

- Babylonische Quellen: 3

- Palästinische Quellen: 9

\section{Jalkut Schimoni Habakuk}

Jalkut Habakuk umfasst knapp 17 hebräische Textseiten und bezieht sich auf die ebenfalls drei Kapitel des Buches Habakuk.

84 bChag 12a; bGit 7a-b; bJoma 21b.

85 GenR 56,5; GenR 63,12; GenR 63,12 (v).

86 Seder Olam 20.

87 TanB bemidbar 16; TanB bemidbar 32; TanB metsora 11.

$88 \operatorname{SDtn} \S 10(\mathrm{v})$.

89 Vgl. TanB bemidbar 2 und Jalkut Schimoni Nahum Kapitel 1. 
Tab. 8: Anzahl der in Jalkut Schimoni Habakuk verwendeten Quellen

\begin{tabular}{llll}
\hline Quelle & Anzahl & Quelle & Anzahl \\
\hline bTalmud $^{90}$ & 12 & Midrasch Samuel $^{91}$ & 2 \\
Mekhilta $^{92}$ & 9 & Unbekannt $^{93}$ & 2 \\
Tanchuma $^{94}$ & 6 & Canticum Rabba $^{95}$ & 1 \\
Midrasch Psalmen $^{96}$ & 5 & Seder Olam $^{97}$ & 1 \\
jTalmud $^{98}$ & 1 & Threni Rabba $^{99}$ & 1 \\
Genesis Rabba $^{100}$ & 3 & & \\
\hline
\end{tabular}

Besonders hervorzuheben sind in diesem Buch die Verbindungen verschiedener Quellen zu einer Auslegung. Die Auslegung beginnt mit Hab 1,7. Die Verse Hab 1,3 und Hab 1,4 werden in Kapitel 3 ausgelegt. Hier werden sie thematisch in die Auslegung eingefügt. Dafür wird folgend auf die Auslegung zu Hab 3,1 (Quelle: MidrPs 90,2), die Auslegung zu Hab 1,3 und Hab 1,4 mit der Quelle MidrPs 90,7 eingefügt, worauf die vorherige Quelle MidrPs 90,2 folgend mit der Auslegung zu Hab 3,1 fortgesetzt wird.

Zur Auslegung von Hab 3,6 wird die Quelle LevR 13,2 ergänzt. Innerhalb der Quelle gibt es keine namentliche Benennung des Berges Moria. Es wird der Berg Sinai in der gleichen Auslegung benannt und um eine weitere Auslegung zum Berg Moria ergänzt. Dieser Textteil ist nicht Teil der Quelle. Zwei Quellen auf die verwiesen wird bleiben ungeklärt. ${ }^{101}$ Auf insgesamt 10 Quellenstücke wird ausschließlich oder textergänzend verwiesen. Die Quellenauswertung ergibt die folgenden Werte:

90 bAZ 3b-4a; bBB 98a; bBer 7b; bBQ 38a; bMeg 28b; bPes 8a; bSanh 7b; bSanh 92a; bSanh 97b; bSchab 149b; bSchab 149b-150a (v); bSota 49a.

91 MidrSam 2,1; MidrSam 4,3 (v).

92 Mek bachodesch 10; Mek bachodesch 10 (v); Mek bachodesch 6; Mek beschallach 4; Mek beschallach 5; Mek beschallach 7; Mek schira 4 (v); Mek schira 6 (v); Mek schira 10 (v).

93 Es handelt sich in beiden Fällen um ungeklärte Verweise.

94 TanB schemini 10; TanB schemot 10; TanB schemot 10 (v); TanB tazri'a 10; TanB teruma 3; TanB tetsaw 10 .

95 CantR 1,14 (v).

96 MidrPs 7,17; MidrPs 22,1; MidrPs 90,2 wird zweimal angeführt; MidrPs 90,7.

97 Seder Olam 20.

$98 \mathrm{jRH} 3,8$.

$99 \operatorname{ThrR} 2,6$.

100 GenR 47,5; GenR 53,3; GenR 68,9.

101 Einmal auf den $\S 20$ oder $\S 30$ (siehe Hab $3 \S 564$ ) und auf $\S 94$. 
- Babylonische Quellen: 12

- Palästinische Quellen: 30

- Unbekannt: 2

\section{Jalkut Schimoni Zephanja}

Jalkut Zephanja umfasst drei Kapitel und neun hebräische Textseiten.

Tab. 9: Anzahl der in Jalkut Schimoni Zephanja verwendeten Quellen

\begin{tabular}{llll}
\hline Quelle & Anzahl & Quelle & Anzahl \\
\hline bTalmud $^{102}$ & 10 & Tanchuma $^{103}$ & 2 \\
Genesis Rabba $^{104}$ & 4 & Midrasch Samuel $^{105}$ & 1 \\
Pesiqta Rabbati $^{106}$ & 3 & Threni Rabba $^{107}$ & 1 \\
Pes. de-Rav Kahana $^{108}$ & 2 & & \\
\hline
\end{tabular}

Die Auslegung des Jalkut beginnt mit Zeph 1,1 und der Quelle bMeg 15a. Es ist dieselbe Quelle, die zur Auslegung von Mal 1,1 in Jalkut Maleachi als erste Quelle verwendet wird. Interessant ist hier, dass die Auslegung zu Zephanja und das Quellenstück zur Auslegung von Maleachi nahtlos aneinander anschließen. Zwischen die Auslegungen von Zeph 1,9 und Zeph 1,10 ist eine Auslegung zu Zeph 1,12 geschaltet. Auf insgesamt 2 Quellenstücke wird ausschließlich oder textergänzend verwiesen. Die Quellenauswertung ergibt die folgenden Werte:

- Babylonische Quellen: 10

- Palästinische Quellen: 13

102 bAZ 24a; bAZ 54b-55a; bBer 28a; bChag 4b; bJeb 63a; bJeb 78b; bMeg 15a; bMeg 15a (v); bSanh 39a; bSchab 139a.

103 TanB metsora 12; TanB noach 19.

104 GenR 28,5; GenR 28,6; GenR 28,7; GenR 66,7.

105 MidrSam 11,5.

106 PR 26,1/2; PR 34,2; PR 8,3-4.

107 ThrR pet. 31.

108 PRK 16,8 (v); PRK 19,1. 


\section{Jalkut Schimoni Haggai}

Jalkut Haggai umfasst zwei Kapitel und vier hebräische Textseiten. Es ist damit neben Jalkut Nahum der kürzeste Kommentar des Zwölfprophetenbuches.

Tab. 10: Anzahl der in Jalkut Schimoni Haggai verwendeten Quellen

\begin{tabular}{llll}
\hline Quelle & Anzahl & Quelle & Anzahl \\
\hline bTalmud $^{109}$ & 3 & Jelamdenu $^{110}$ & 1 \\
Sifre Dtn $^{111}$ & 2 & Levitikus Rabba $^{112}$ & 1 \\
Tanchuma $^{113}$ & 2 & Mekhilta $^{114}$ & 1 \\
\hline
\end{tabular}

In Kapitel 1 wird der Vers Hag 2,23 folgend auf Hag 1,1 angeführt, wobei beide Verse nicht an dieser Stelle ausgelegt werden, sondern auf die jeweiligen Auslegungen verwiesen wird. Dies ist ein interessanter Fakt, der noch einmal zeigt, dass der Autor für eine passende inhaltliche Auslegung auch die Reihenfolge der auszulegenden Verse unterbricht. Gerade an dieser Stelle hätte der Autor den Vers auch mit dem entsprechenden Verweis an die korrekte Stelle in Kapitel 2 einflechten können, vor allem, weil es keinen literarischen Bruch gibt, da in Haggai eben „nur“ auf die Auslegung verwiesen wird. Der Autor entscheidet sich also bewusst für eine „nicht fortlaufende Auslegung“ zugunsten der stimmigen thematischen Folge.

In Kapitel 2 wird die Auslegung zu Hag 2,8 nicht nur wie angegeben aus bNid 69b entnommen, sondern auch aus bNid 70b-71a. Diese Auslegung wird aus allen drei Kapiteln der Quellen zusammengeschnitten. Auf insgesamt 3 Quellenstücke wird ausschließlich oder textergänzend verwiesen. Die statistische Quellenauswertung ergibt die folgenden Werte:

- Babylonische Quellen: 3

_ Palästinische Quellen: 7

109 bNid 71a; bPes 16b-17a; bJoma 21b.

110 Es handelt sich um einen Textverweis.

$111 \operatorname{SDtn} \S 27 ; \operatorname{SDtn} \S 27(\mathrm{v})$.

112 LevR 81.

113 TanB bechuq 3; TanB qedoschim 7.

114 Mek bachodesch 1 (v). 


\section{Jalkut Schimoni Sacharja}

Jalkut Sacharja ist neben Jalkut Hosea einer der längsten Kommentare. Er umfasst dreizehn Kapitel und knapp 42 hebräische Textseiten. Eine Besonderheit des Sacharjakommentars ist, dass dieser keine Auslegung zu Sach 7 aufweist. Das Phänomen, ein Kapitel nicht zu kommentieren, findet sich auch in Jalkut Nahum.

Tab. 11: Anzahl der in Jalkut Schimoni Sacharja verwendeten Quellen

\begin{tabular}{llll}
\hline Quelle & Anzahl & Quelle & Anzahl \\
\hline bTalmud $^{115}$ & 49 & Jelamdenu & 2 \\
Tanchuma $^{116}$ & 9 & Mekhilta & 117 \\
Genesis Rabba $^{118}$ & 8 & Unbekannt $^{119}$ & 2 \\
Midrasch Psalmen $^{120}$ & 4 & Abot de-Rabbi Natan $^{121}$ & 1 \\
Pirk. de-Rab. Eliezer $^{122}$ & 4 & Mischna $^{123}$ & 1 \\
Levitikus Rabba $^{124}$ & 3 & Sifra $^{125}$ & 1 \\
Pes. de-Rab. Kahana $^{126}$ & 3 & Threni Rabba $^{127}$ & 1 \\
\hline
\end{tabular}

115 bAZ 4a; bAZ 10b (v); bBB 12b; bBB 75b; bBB 75b (v); bBer 51a; bBer 55b; bBer 56b; bBQ 60b; bChag 10a; bChul 56b; bChul 63a; bChul 92a-b; bEr 21a (v); bHor 13a (v); bJoma 39b; b Joma 69b (v); bJoma 86a; bMak 22a-b; bMak 24a-b (v); bMeg 3a; bPes 50a wird dreimal angeführt; bPes 68a; bRH 16b-17a; bRH 18b; bSanh 6b-7a; bSanh 24a wird zweimal angeführt; bSanh 24a (v); bSanh 93a wird zweimal angeführt; bSanh 93a (v); bSanh 98a wird zweimal angeführt; bSanh 103b; bSanh 103b (v); bSanh 105a (v); bSchab 32b; bSchebu 38b-39a; bSota 48b-49a; bSuk 5a (v); bSuk 52a; bSuk 52b; bTaan 3a-b; bTaan 9a; bTaan 9b; bZeb 54b.

116 TanB achare 18; TanB emor 18; TanB metsora 10; TanB qedoschim 7 (v); TanB schoftim 10; TanB tazri'a 16; TanB toldot 20; TanB tsaw 4 (v); TanB wa-jischlach 30.

117 Mek schira 6 (v) wird zweimal angeführt.

118 GenR 12,6 (v); GenR 14,4; GenR 42,3 (v); GenR 56,11; GenR 56,9; GenR 70,6; GenR 70,6 (v); GenR 75,1 .

119 Bei diesen beiden unbekannten Quellenstücken handelt es sich um Stellen, die der Quelle PR zugeteilt werden aber nicht näher bestimmt sind. Es handelt sich einmal um einen Verweis und zum zweiten um die Stelle selbst. Es ist also ein interner Verweis. Siehe Sacharja § 569/§ 574. Außerdem siehe Kapitel 6.3 „Interne Verweise“.

120 MidrPs 12,5; MidrPs 15,2; MidrPs 38,1; MidrPs 119,2.

121 ARN A 4.

122 PRE 28; PRE 31; PRE 36; PRE 38 (v).

123 mAbot 1,18 .

124 LevR 31,4; LevR 6,3 wird zweimal angeführt.

125 Sifra tsaw per. $18,1$.

126 PRK 4,7; PRK 20,7 (v) wird zweimal angeführt.

127 ThrR 2,8; ThrR 4,14. 


\begin{tabular}{|c|c|c|c|}
\hline Quelle & Anzahl & Quelle & Anzahl \\
\hline Pesiqta Rabbati ${ }^{128}$ & 3 & Tosefta $^{129}$ & 1 \\
\hline Sifre $\operatorname{Dtn}^{130}$ & 3 & & \\
\hline
\end{tabular}

Jalkut Sacharja weist im Vergleich zu den vorausgegangenen Schriften mehrere Besonderheiten auf, die sich auf Verweise innerhalb des Zwölfprophetenbuches beziehen. Dies ist vermutlich auch mit der Stellung dieses Buches als vorletzte Auslegung des Zwölfprophetenbuches zu begründen. Es scheint, als seien viele Themen zu diesem Zeitpunkt besprochen und man verweist an einigen Stellen nur noch auf bereits geführte Auslegungen. Kapitel 7 wird im Jalkut überhaupt nicht ausgelegt. Es wird von Kapitel 6 direkt zum Kapitel 8 übergegangen.

In Jalkut Sacharja wird Sach 1,8 im Kapitel 1 und im Kapitel 6 ausgelegt. Im Kapitel 1 wird neben der dort geführten Auslegung zusätzlich auf die Auslegung in Kapitel 6 verwiesen. Dies zeigt, dass die geführte Auslegung Relevanz für das 1. und das 6. Kapitel im Buch Hosea hat.

Zum bereits angesprochenen Quellenabschnitt PR zu Beginn von Kapitel 6 noch eine weitere Besonderheit: Hier wird die fortlaufende Auslegung mehrfach gebrochen: Nach der Auslegung bis Sach 6,3 folgen drei Auslegungen zu Sach 6,2, darauf dann fortlaufende Auslegungen bis Sach 6,4. Dann folgt der besprochene Exkurs zu Sach 1,8, der Sprung zu Sach 6,7 und der weitere Sprung zurück zu Sach 6,1, mit dem das sechste Kapitel beendet wird, auf welches direkt das 8. Kapitel folgt. Zwei Quellenstücke aus der Quelle PR können nicht vollständig bestimmt werden und bleiben deshalb ungeklärt. Auf insgesamt 24 Quellenstücke wird ausschließlich oder textergänzend verwiesen. Die Quellenauswertung ergibt die folgenden Werte:

- Babylonische Quellen: 49

- Palästinische Quellen: 46

- Unbekannt: 2

128 PR 35,2; PR 39,1; PR 8,4.

129 tSota $11,14$.

$130 \operatorname{SDtn} \S 1 ; \operatorname{SDtn} \S 10(\mathrm{v}) ; \operatorname{SDtn} \S 77$. 


\section{Jalkut Schimoni Maleachi}

Jalkut Schimoni Maleachi umfasst drei Kapitel und 24 hebräische Textseiten.

Tab. 12: Anzahl der in Jalkut Schimoni Maleachi verwendeten Quellen

\begin{tabular}{llll}
\hline Quelle & Anzahl & Quelle & Anzahl \\
\hline bTalmud $^{131}$ & 29 & Pes. de-Rab. Kahana & 1 \\
Tanchuma $^{133}$ & 7 & Pesiqta Rabbati $^{134}$ & 1 \\
Genesis Rabba $^{135}$ & 6 & Pirk. de-Rab. Eliezer & 1 \\
Midrasch Psalmen $^{137}$ & 4 & Rut Rabba & 138 \\
Sifra $^{139}$ & 4 & Seder Elijahu Rabba & 1 \\
Levitikus Rabba $^{141}$ & 3 & Sifre Dtn & 1 \\
Mekhilta $^{143}$ & 3 & Sifre Numeri & 1 \\
Abot de-Rabbi Natan $^{145}$ & 2 & Unbekannte Quelle & 1 \\
Mischna $^{146}$ & 1 & & \\
\hline
\end{tabular}

In Kapitel 2 wird zur Auslegung von Mal 2,8, folgend auf die Quelle bBek 26b, eine unbekannte Quelle angeführt. Sie zeigt eine weitere Auslegung und Ähnlichkeiten zu

131 bBB 16b; bBB 60b; bBB 60b (v); bBek 26b; bChag 15b; bChag 5a; bChag 9b; bGit 90b; bMeg 15a; bMen 110a; bNed 8b; bQid 40a (v); bQid 71a; bRH 16b-17a (v); bSanh 6b; bSanh 82a; bSanh 96a (v); bSanh 110b; bSchab 32b; bSchab 63a; bSchab 89a; bSchab 118a; bSchab 151b; bSuk 50a; bTaan 20a; bTaan 8b; bTaan 9a.

132 PRK 24,15 (v).

133 TanB achare 14; TanB achare 14 (v); TanB naso 8 (v); TanB nitsabim 1 (v); TanB schelach; TanB teruma 8; TanB wa-jiggasch 11.

134 PR 4 (v).

135 GenR 18,5; GenR 6,6; GenR 68,10; GenR 80,7; GenR 80,7 (v); GenR 85,1.

136 PRE 43.

137 MidrPs 1,16; MidrPs 31,9; MidrPs 43,1; MidrPs 9,9.

138 RutR 5,4 (v).

139 Sifra schemini Mek deMilluim 31; Sifra schemini Mek deMilluim 37; Sifra schemini Mek deMilluim 38; Sifra tsaw per. 16,10.

140 SER [15].

$141 \operatorname{LevR}$ 7,3 (v); LevR 34,8; LevR 34,8 (v).

$142 \operatorname{SDtn} \S 306$.

143 Mek bo pischa 12 (v); Mek schira 1; Mek schira 6 (v)

144 SNum $\S 119$.

145 ARN 3,6 (v); ARN 12.

$146 \operatorname{mEd} 8,7$. 
SNum $\S 67$ und TanB beha'alotkha 13, kann aber nicht einer bestimmten Quelle zugeordnet werden.

In Jalkut Maleachi können eher marginale aber nicht uninteressante Abweichungen in der Reihenfolge der kommentierten Verse von Maleachi beschrieben werden: Im zweiten Kapitel springt die Auslegung von Mal 2,12 zurück auf 2,11, um dann mit Mal 2,13 fortzufahren. Interessant ist, dass der abweichende Abschnitt mit der neuen Quelle GenR 85,1 an diese Stelle eingefügt wird. Auch hier handelt es sich um eine bewusste Entscheidung, da die Quelle auch fortlaufend richtig gereiht, vor die Quelle bSanh 82a hätte gesetzt werden können. Die gleiche Situation zeigt sich in Kapitel 3. Dort wird mit der neuen Quelle GenR 6,6 eine weitere Auslegung zu Mal 3,19 angeführt, nachdem bereits Auslegungen zu Mal 2,20 geführt wurden.

Ein Unterschied zeigt sich in Bezug auf die zwei Beispiele: Während im Kapitel 2 zwei Versteile, die bereits in der fortlaufenden Auslegung erwähnt werden erneut nachträglich ausgelegt werden, wird in Kapitel 3 mit dem Versteil aus Mal 3,19 ein noch nicht ausgelegter Teil des Verses nachträglich ausgelegt.

Auf insgesamt 17 Quellenstücke wird ausschließlich oder textergänzend verwiesen. Die statistische Quellenauswertung ergibt die folgenden Werte:

- Babylonische Quellen: 29

- Palästinische Quellen: 42

- Unbekannt: 1

\section{Jalkut Schimoni zum Zwölfprophetenbuch - Gesamtauswertung}

Die zwölf Einzelauswertungen der Verwendung der Quellen in Jalkut Schimoni zu den zwölf Propheten können in drei Kategorien unterteilt werden: Die erste Gruppe sind Schriften, deren Quellenverhältnis zwischen babylonischen und palästinischen Quellen klar ausgeglichen ist. Dazu gehören die Kommentare zu Amos, Obadja, Zephanja und Sacharja. Die zweite Gruppe bilden die Kommentare, bei denen die palästinischen Quellen leicht überwiegen. Dazu gehören die Schriften Hosea, Micha, Habakuk und Maleachi. Die letzte Gruppe zeigt die Kommentare, bei denen die palästinischen Schriften deutlich überwiegen. Diese sind Joel, Jona, Nahum, Haggai. Von den insgesamt 610 Quellenstücken im Jalkut Schimoni zum Zwölfprophetenbuch sind zu verzeichnen:

- Babylonische Quellen: 247

- Palästinische Quellen: 352

- Unbekannt: 11 
Mit der Auszählung der Quellenstücke kann nicht das Längenverhältnis der einzelnen Quellenstücke zueinander erfasst werden. So sind beispielsweise in Jalkut Haggai mehr kurze palästinischen Quellenstücke verwendet worden, dies gleicht die Länge der Quellenstücke des babylonischen Talmuds aber wieder aus.

Jalkut Jona wurde bereits für seine besondere narrative Struktur hervorgehoben. Auch wurde darauf hingewiesen, dass zu Jona ein fortlaufender Midrasch existiert, auf den der Autor des Jalkut zurückgreifen konnte. Umso interessanter ist, dass der Jona-Midrasch nur in Teilen benutzt wurde und sowohl von weiteren Midraschim ergänzt, als auch mit vier Quellenstücken des babylonischen Talmuds erweitert wird. Mehr als 50\% des Gesamttextes in Jona werden von den Quellen PRE und Mekhilta abgedeckt. Der Midrasch Jona steht im quantitativen Textverhältnis mindestens gleichwertig zu PRE.

Der babylonische Talmud ist die einzige Quelle, die in jedem der zwölf Kommentare als Quelle verwendet wird. Als Einzelquelle ist der Babylonische Talmud klar als Hauptquelle zu identifizieren. Dies wiederspricht der Annahme Meyers, Genesis Rabba sei Hauptquelle des Jalkut und strukturgebend für den Jalkut. ${ }^{147}$

Neben dem Babylonischen Talmud kann Tanchuma als eine Schrift angeführt werden, die außer in Jona, in allen Schriften zur Kommentierung verwendet wird. Die Quellenstücke aus Tanchuma zeichnen sich außerdem in der Regel durch ihre besondere Länge aus.

Die Verhältnisse der palästinischen Quellen können weiter differenziert werden. So setzen diese sich aus den verschiedensten Midraschim unterschiedlicher Entstehungszeiträume und dem Jerusalemer Talmud zusammen: 352 Quellenstücke der palästinischen Tradition können wie folgt weiter differenziert werden. Von 352 Quellenstücken entfallen:

- 125 auf frühe und halachische Midraschim. Dabei entfallen 60 auf GenR; 7 auf

ThR; 29 auf Mekhilta; 21 auf SDtn; 6 auf Sifra; und 2 auf SNum

- 53 Quellenstücke stammen aus Tanchuma

- 29 Quellenstücke stammen aus der Rabbot Literatur. Dabei entfallen 3 auf

CantR; 21 auf LevR; 2 auf RutR; 3 auf DtnR

- 25 Quellenstücke stammen aus MidrPs

- 15 entstammen aus Jelamdenu; 20 aus PR; und 29 aus PRK

- 15 stammen aus dem Jerusalemer Talmud

Die Quelle Jelamdenu wird von insgesamt 610 Quellenstücken „nur“ 15 Mal verwendet. Literaturwissenschaftlich wird diese Quelle, die nur fragmentarisch überliefert ist, in einer sehr engen textuellen Verwandtschaft zur Quelle Tanchuma gesehen. So

147 Vgl. David Théophile Ivan Meyer, La Nature Éditoriale du Yalkut Shimoni. Une étude exploratoire des citations de Bereshit Rabbah XXII,8-10 dans le Yalkut Shimoni (PhD, Universität Leuven), 4-5. 
werden beide Texte im Mittelalter häufig als Synonym für den jeweils anderen angeführt. ${ }^{148}$ Der Jalkut unterscheidet klar zwischen Tanchuma und der Quelle Jelamdenu. Tanchuma und Jelamdenu werden zu den sogenannten Homilien-Midraschim gezählt. Somit wird vermutet, dass diese Gattung der Midraschim als Art Predigtvorlage oder als eine Verschriftlichung gehaltener Predigten gilt. ${ }^{149} \mathrm{Ob}$ es sich tatsächlich um Predigten handelt, bleibt offen, deutlich ist jedoch, dass der Jalkut beide Werke differenziert und Tanchuma der Quelle Jelamdenu deutlich vorzieht. Deutlich wird außerdem, dass mit 53 Stücken aus Tanchuma und weiteren 15 Stücken aus Jelamdenu, sowie 65 Stücken anderer Homilien-Midraschim (PR, PRK, LevR, DtnR) der literarische Charakter einer Predigt im Jalkut hervorgehoben wird.

Während die früheren Werke der Rabbot Literatur (GenR, ThrR als älteste; CantR, LevR, RutR und DtnR, alle vor dem 11. Jh. datiert) im Jalkut Schimoni zum Zwölfprophetenbuch verwendet werden, wird ExR und NumR nicht zitiert. Die Datierung von ExR ist weiter umstritten, sie wird ins 12.-13. Jh. angesetzt. ${ }^{150}$ NumR, der ursprünglich aus zwei Teilen bestand, wird als vollständiger Midrasch ins 12. Jh. datiert. ${ }^{151}$ Inwieweit die Nicht-Verwendung von NumR und ExR Aussagen zur Datierung von Jalkut Schimoni zum Zwölfprophetenbuch erlauben, ist nicht abschließend zu klären. Zunächst spricht diese Auswahl für eine Datierung des Jalkut, die diesen beiden Quellen vorausgeht. Ebenso könnten aber auch inhaltliche Faktoren ein Grund dafür sein, dass diese nicht verwendet wurden.

148 Günter Stemberger, Einleitung in Talmud und Midrasch, 337.

149 Doris Lenhard, Die rabbinische Homilie: ein formanalytischer Index (Frankfurt: Gesellschaft zur Förderung Judaistischer Studien, 1998), 6-8.

150 Vgl. Günter Stemberger, Einleitung in Talmud und Midrasch, 341-342.

151 Vgl. Günter Stemberger, Einleitung in Talmud und Midrasch, 343-344. 